\title{
Logical Design of Quaternary Signed Digit Conversion Circuit and its Effectuation using Operational Amplifier
}

\author{
Tanay Chattopadhyay and Tamal Sarkar
}

\begin{abstract}
In binary number system carry is a major problem in arithmetical operation. We have to suffer $O(n)$ carry propagation delay in $n$-bit binary operation. To overcome this problem signed digit is required for carry free arithmetical operation. Further, literature reviews suggest that multi-valued logic (MVL) would be a better choice to address the problem of developing faster chips for performing faster computational operation. Quaternary Signed Digit $(Q S D)$ have a major contribution in higher radix $(=4)$ carry free arithmetical operation. For digital implementation, the signed digit quaternary numbers are represented using 3-bit 2 's compliment notation. In this paper, a simple and new technique of binary (2's compliment) to QSD conversion is proposed and described. Well-known operational amplifier (OPAMP) based digital to analog converter circuit is also given to verify the above technique.
\end{abstract}

Keywords---Multi-Valued Logic, Binary 2's Compliment Number, Quaternary Signed Digit, Radix Conversion, OPAMP

\section{INTRODUCTION}

$\mathrm{M}$ ODERN computers are based on binary number system (radix $=2$ ). It has two logical states ' 0 ' and ' 1 '. In such system, ' 1 ' plus ' 1 ' is ' 0 ' with carry ' 1 ' (i.e. $1+1=10$ ). This carry should have to add with another ' 1 ', as a result further carry ' 1 ' generates. This creates the delay problem in computer circuits. So to get rid of this carry formation again and again signed digit is essential. In high-speed arithmetical calculation, carry free adders improves the operational performance. Binary logic is restricted to only two logical states; Multi-Valued Logic (MVL) replaces these with finite and infinite numbers of values [1]. Multi-valued logic is a higher radix $(R>2)$ logic system. Non-binary data requires less physical storage space than binary data [2-4]. Depending upon the radix number $\mathrm{R}$, the number system are named as ternary $(\mathrm{R}=3)$, quaternary $(\mathrm{R}=4)$ etc. Ternary logic is based on ternary number system. They can further be divided into two groups; symmetric ternary $\{\overline{1}, 0,1\}$ and ordinary ternary $\{0$,

Tanay Chattopadhyay, Mechanical Operation (Stage-II), Kolaghat Thermal Power Station, WBPDCL, Mecheda, Purbamedinipur, KTPP Sub Post Office, 721137, West Bengal, India. E-mail: tanay2222@rediffmail.com).

Tamal Sarkar, University Science Instrumentation Centre, University of North Bengal, Siliguri, 734013, India. E-mail: tamalsarkar@sify.com
$1,2\}$. Both groups are important in logical and arithmetical operations [5-8]. Quaternary logic is based on radix-4 number system. In quaternary system, the positive integer set $\{0,1,2$, $3\}$ is called ordinary quaternary digit (OQD) and the set of both positive and negative integer $\{\overline{3}, \overline{2}, \overline{1}, 0,1,2,3\}$ is called quaternary signed digit (QSD). Where $\overline{3}=-3, \overline{2}=-2, \overline{1}$ $=-1$. In signed digit representation QSD number can be written as [9]:

$$
X=\sum_{i=0}^{n-1} x_{i} \cdot 4^{i} ; \text { where } x_{i} \in\{\overline{3}, \overline{2}, \overline{1}, 0,1,2,3\}
$$

Signed digit representation is essentially required for carry free arithmetic operation. As such, binary to quaternary signed digit conversion is very much interesting and required topics. This is called 'radix conversion'. The importance of radix conversion is shown in flexogram at fig.1.

Ordinary quaternary number can be easily expressed as two bit binary number [10-15]. The implementation of multi-

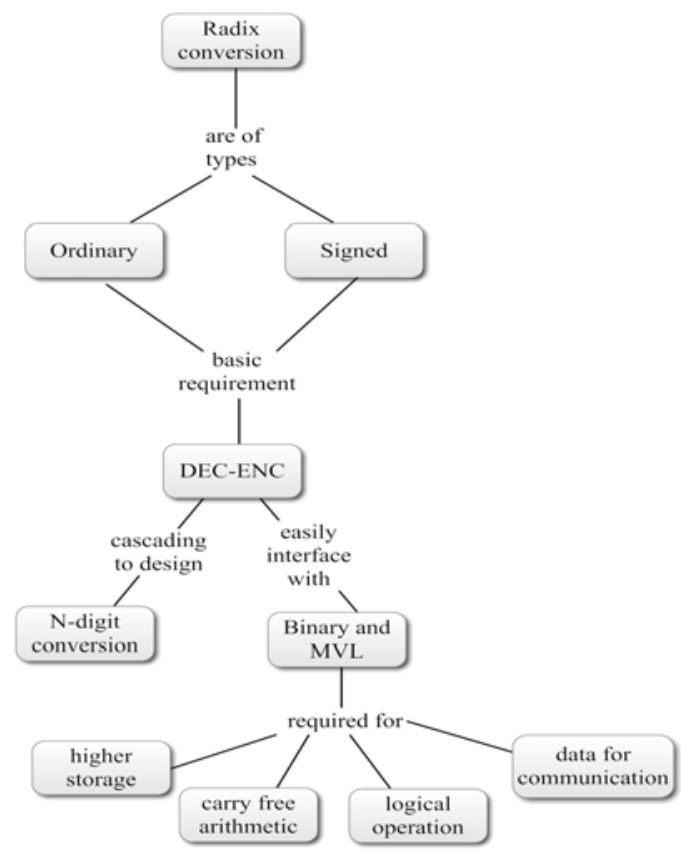

Figure 1: The Importance of Radix Conversion 
valued logic using ordinary quaternary digit has been studied by using various devices, such as $I^{2} \mathrm{~L}[16]$, CCD $[10,17]$,

CMOS [11, 13-14, 18], ECL [12, 19], v MOS [20], MESFET [21] in electronics. In these literatures many logic levels had been considered. Mingoto Jr. proposed quaternary logic circuit using traditional TTL logic family where $0 \mu \mathrm{A}$, $10 \mu \mathrm{A}, 20 \mu \mathrm{A}$ and $30 \mu \mathrm{A}$ are designated as four logic level [22]. Park et al [20] designed quaternary logic circuit using $v$ MOS where $0.5 \mathrm{~V}, 1.5 \mathrm{~V}, 2.5 \mathrm{~V}$ and $3 \mathrm{~V}$. Logic levels in Shanbhag et al are $0 \mathrm{~V}, 2 \mathrm{~V}, 4 \mathrm{~V}$ and $6 \mathrm{~V}$ respectively [13]. Tanable et al [23] designed redundant MVL circuit using CMOS where they use binary two states $0-0,0-1,1-0$ and $1-1$ as quaternary 0, 1, 2 and 3 states respectively. Datla et al [24] designed quaternary cell based on supplementary symmetrical logic circuit structure (SUSLOC) using FET. They used 0V, $1.1 \mathrm{~V}, 2.2 \mathrm{~V}$ and 3.3V for four logical states. Lee et al [25] also designed and simulated 4-valued memory with double-gate thin film transistor. Aragaki et al [26] implemented high density multiple-valued content-addressable memory (MVCAM) where $0 \mathrm{~V}, 1.4 \mathrm{~V}, 2.8 \mathrm{~V}$ and $4.2 \mathrm{~V}$ are considered as different quaternary logic levels. Khader and Siy proposed multi-valued $\mathrm{AB}^{2}$ circuit using $v$ MOS where they also used different voltage levels as quaternary logical states [27-28]. Also some quantum quaternary logic circuits have been proposed by Khan et al [29-30]. To represent 1-digit QSD three bit binary (2's compliment form) is required [31-32]. Although there have many circuits to represents binary to ordinary quaternary conversion in electronics, but no circuit have not been yet proposed to convert binary to QSD number. In this paper, we have mentioned a technique of this conversion as well as proposed a simple technique of $n$-bit binary-signed number (2's compliment) to QSD number system. We have also designed a prototype of digital to analog converter circuit using operational Amplifier (OPAMP) to verify the technique.

\section{TECHNIQUE OF CONVERSION FROM BINARY NUMBER TO QSD NUMBER}

1-digit QSD can be represented by one 3-bit binary equivalent as follows [15]:

$$
\begin{aligned}
& \overline{3}=101 \\
& \overline{2}=110 \\
& \overline{1}=111 \\
& 0=000 \\
& 1=001 \\
& 2=010 \\
& 3=011
\end{aligned}
$$

So to convert $n$-bit binary data to its equivalent $q$-digit QSD data, we have to convert this $n$-bit binary data into $3 q$-bit binary data. To achieve the target, we have to split the $3^{\text {rd }}, 5^{\text {th }}$, $7^{\text {th }}$ bit.... i.e. odd bit (from the LSB to MSB) into two portions [34]. But we cannot split the MSB. If the odd bit is 1 then, it is split into $1 \& 0$ and if it is 0 then, it is split into $0 \& 0$. An example makes it clear, the splitting technique of a binary number $(1101101)_{2}$ is shown below:

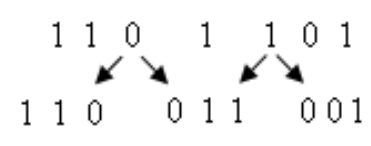

So we have to split the binary data $(q-1)$ times (as example, for conversion of 2-bit quaternary number, the splitting is 1 time; for converting 3-digit quaternary number the split is 2-times and so on). In each such splitting one extra bit is generated. So, the required binary bits for conversion to it's QSD equivalent $(n)=$ (Total numbers of bits generated after divisions) - (extra bit generated due to splitting).

$$
\begin{aligned}
n & =3 q-\{1 \times(q-1)\} \\
& =(2 q+1)
\end{aligned}
$$

So, number of bits of the binary number should be $3,5,7$, 9 etc for converting it to its equivalent QSD number. Now every 3-bit can be converted to its equivalent QSD according to the equation (2). The following two examples as given below will help to make the things clear.

1) Let $(-155)_{10}=(101100101)_{2}$ have be converted to its equivalent QSD. '(101100101) $)_{2}$ ' is 9-bit binary data. Its $3^{\text {rd }}$ bit is $1,5^{\text {th }}$ bit is 0 and $7^{\text {th }}$ bit is 1 . So from the equation (3) we can say that, its QSD equivalent is of 4-digit. Hence according to the splitting technique stated above the binary data can be expressed as follows.

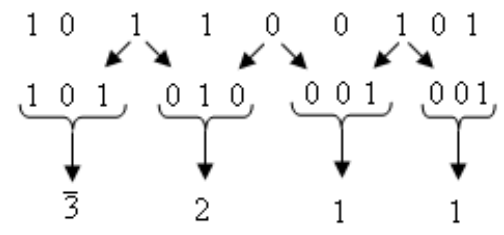

So the QSD equivalent of $(101100101)_{2}$ is $(\overline{3} 211)_{4}$.

2) Let $(49)_{10}=(0110001)_{2}$ is to be converted to its equivalent QSD. '(0110001), is 7-bit binary number. According to the previous discussion the conversion is as follows

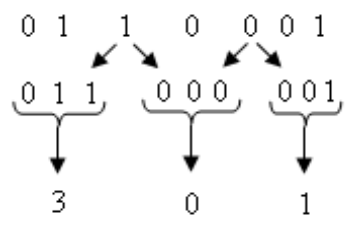

So the QSD equivalent of $(110001)_{2}$ is $(301)_{4}$.

III. VerificAtion OF the Above TEChNique Using ELECTRONIC CIRCUITS 


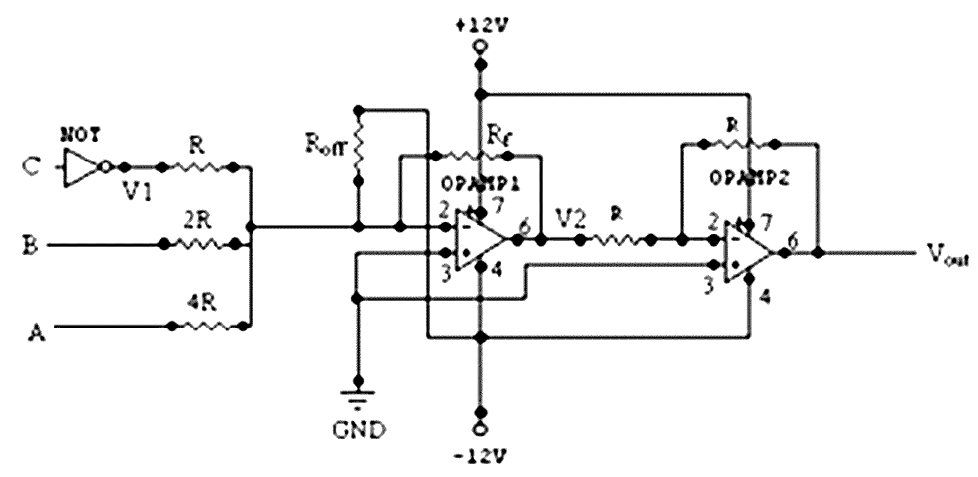

Figure 2(a): Single-Digit QSD Converter Circuit

It is possible to design One-digit QSD converter by using operational amplifier (OPAMP) [35-36]. To implement the design, we have to select some voltage level for QSD. In present study, we have selected the following voltage level for QSD: $-\overline{3} \approx-3$ volts, $\overline{2} \approx-2$ volts, $\overline{1} \approx-1$ volts, $0 \approx 0$ volts, $1 \approx 1$ volts, $2 \approx 2$ volts and $3 \approx 3$ volts respectively. For design $\&$ implementation purpose, we have made some modification in the common circuit of digital to analog converter using OP-

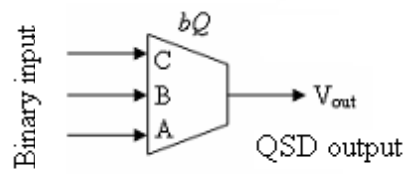

Figure 2(b): Block Diagram of the Single Digit QSD Converter Block (bQbock)

AMP to get 'Single-digit QSD converter'. The electronic circuit for Single-digit QSD converter is shown in the fig-2(a) and the corresponding logic table with analog values were given in Table 1. In this design, we have used two operational amplifiers (OPAMP). For experimental setup, we have used the IC 741 (OPAMP) and IC 74LS04 (NOT GATE). OPAMP1 acts as 3-bit digital-to-analog (D/A) converter and OAMP2 acts as inverting gate. The inputs $\mathrm{C}, \mathrm{B}, \mathrm{A}$ are the binary (2's compliment) with logical state 1 (dc voltage range of 3.5 to 5 volt) or 0 (dc voltage range 0 to 1 volt) where input $\mathrm{C}$ is the most significant bit (MSB) and $\mathrm{A}$ is the least significant bit (LSB). C is connected to the input of the OPAMP1 through binary NOT gate. When $\mathrm{C}=0$ volt then voltage at V1 point is 5 volt (experimental measured value is 4.42 volt) and when $C=5$ volt then voltage at $V 1$ point is 0 volt (experimental measured value is 0.11 volt) i.e. we can say that inputs of the $\mathrm{D} / \mathrm{A}$ converter are $\overline{\mathrm{C}}, \mathrm{B}$, A. If the inputs $(\mathrm{C}, \mathrm{B}, \mathrm{A})$ are of logical state 000 then, actually 100 (equivalent to 4 in decimal) is fed to the input of the D/A converter unit. To get logic output ' 0 ', we need to put an offset from -12 volt through resistance $R_{\text {off }}$ according to the fig-2(a). The block diagram of this binary to QSD converter (bQ-block) circuit is shown in the fig-2(b).

The offset voltage produces in the circuit is

$$
-\frac{R_{f}}{R_{\text {off }}}(-12)=4
$$

Hence, the output voltage of the A/D converter circuit (V2 point) is [29],

\begin{tabular}{ccc|ccccc}
\hline \hline \multicolumn{3}{c|}{$\begin{array}{c}\text { Inputs } \\
\text { (Volt) }\end{array}$} & \multicolumn{5}{c}{ Outputs $\left[\mathrm{V}_{0}\right]$ (Volts) } \\
\hline $\mathrm{C}$ & $\mathrm{B}$ & $\mathrm{A}$ & Ideal & Calculated & Experimental & Error $_{1}$ & Error $_{2}$ \\
\hline 5 & 0 & 5 & -3 & -2.79 & -2.86 & 0.07 & -0.14 \\
5 & 5 & 0 & -2 & -1.77 & -1.67 & 0.10 & -0.33 \\
5 & 5 & 5 & -1 & -0.74 & -0.45 & 0.29 & -0.26 \\
0 & 0 & 0 & 0 & 0.28 & -0.09 & 0.37 & 0.09 \\
0 & 0 & 5 & 1 & 1.31 & 1.31 & 0.00 & 0.31 \\
0 & 5 & 0 & 2 & 2.33 & 2.40 & 0.07 & 0.40 \\
0 & 5 & 5 & 3 & 3.57 & 3.53 & 0.04 & 0.53 \\
\hline \hline \multicolumn{7}{c}{$-\left(\frac{R_{f}}{L} \overline{L S_{C}}+\frac{R_{f}}{2 R} L S_{B}+\frac{R_{f}}{4 R} L S_{A}\right)+\frac{12 \cdot R_{f}}{R_{\text {off }}}$} & $(5)$
\end{tabular}

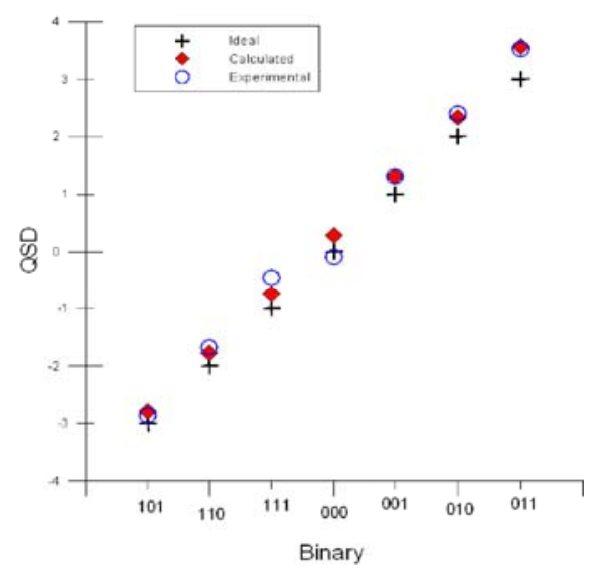

Figure 3: Experimental, Theoretical and Ideal QSD values for Different Binary Inputs 
where $L S_{C}, L S_{B}$ and $L S_{A}$ are the logical states of the input C, B and A respectively. Using $R_{f}=820 K \Omega, R=1 M \Omega$ and calculating from the equation (4) $R_{\text {off }}=2.4 M \Omega$ (in experiment we use $R_{\text {off }}=(2.2 M \Omega+330 K \Omega+47 K \Omega)=$ $2.577 \mathrm{M} \Omega$ ). In the experimental setup we find the voltage for binary 'logical-1' state is 5.08 volt. Hence putting these values of resistance and experimentally measured voltage, we found the following results as shown in Table 1. Fig. 3 shows the experimental, theoretical and ideal QSD values for different binary inputs. The graphical representation of the output voltage with time is also shown in the Fig 4 . From table- 1 we see that there are two types of errors, one is difference between experimental results and theoretical value (we define it Error ${ }_{1}$ ) and other is difference between experimental and ideal one (we defined it as Error $_{2}$ ).

$$
\begin{aligned}
& \text { Error }_{1}=\mid \text { Experimental }- \text { theoritical } \mid \\
& \text { Error }_{2}=(\text { Ideal }- \text { Experimental })
\end{aligned}
$$

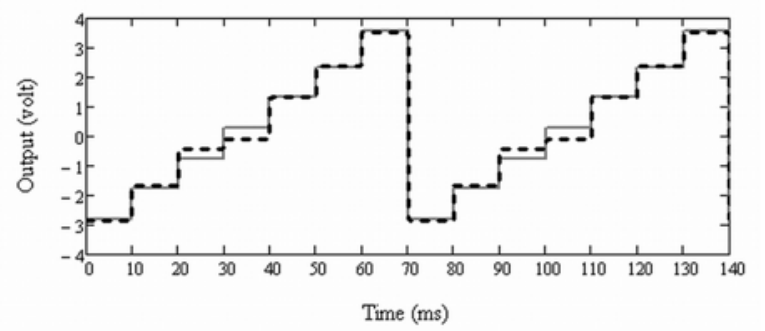

Figure 4: Output waveform of Binary to QSD Converter Circuit. Solid Line: Calculated Result, Dotted Line: Experimental Result

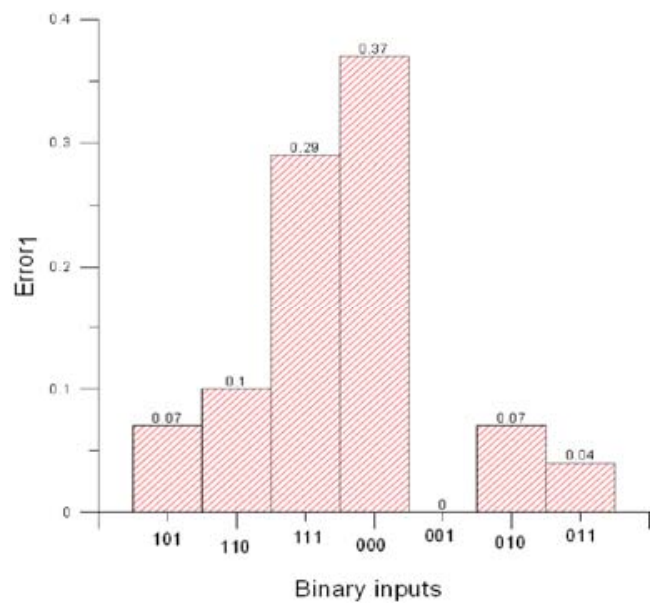

Figure 5(a): Error ${ }_{1}$ Detected in Experiment and Theory for Different set of Binary Inputs

These errors are plotted for different logical binary inputs in Fig. 5(a) and 5(b) respectively. Here we see that errors become small for positive QSD numbers than negative QSD numbers.

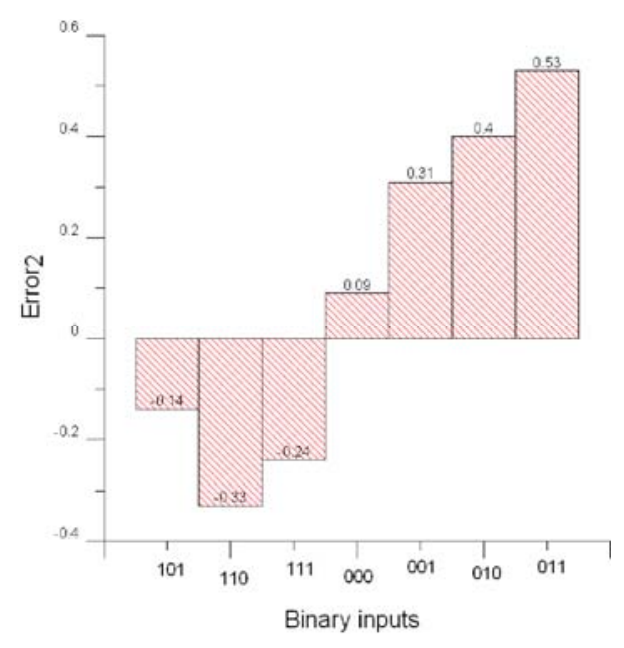

Figure 5(b): Error $_{2}$ found in Experiment and Ideal QSD values for Different set of Binary Inputs

Binary to QSD conversion by electronic circuit is not so easy, because in ordinary quaternary conversion required only 4 states, but in QSD conversion we required three extra logical states. To represent these states, voltage level selection is important and the difference between two successive states should be distinguishable with precision. Otherwise, data error may occur. The sources of error are noises present in the components used to design the circuit. The noise also depends on frequency of operation. Noise minimization is an important factor in this regards. OPAMP operates in high voltage span $-12 \mathrm{~V}$ to $+12 \mathrm{~V}$, but the power dissipation is high enough in respect to CMOS based MVL circuits [37-38]. It may operate at the frequency $2.2 \mathrm{MHz}$. One the other hand our proposed circuit can be designed with $v$ MOS- OPAMP which has greatly enhanced the accuracy of analog computation. Namely, the output offset voltage appearing in a multi-valued logic gate does not increase with the increase in the number of input terminals and the output offset does not propagate to the next stage, thus making it very promising for implementing multi-valued logic integrated circuits [39].

\section{CONCLUSION AND DisCUSSION}

In this paper, we have reported a new and easy method for conversion from binary number (2's compliment representation) to quaternary-signed digit (QSD). Some important issues as follows are discussed. The above techniques can be easily used for designing $n$-bit binary $\left(a_{n} a_{n-1} \cdots a_{2} a_{1} a_{0}\right)_{2}$ to $q$-digit QSD data $\left(m_{q} m_{q-1} \cdots m_{2} m_{1} m_{0}\right)_{4}$ with the help of single bit QSD converter unit (bQ-block). The corresponding circuit is shown in the fig- 6 . Here binary bits $a_{n}, a_{n-1}$ and $a_{n-2}$ are connected with three inputs ' $\mathrm{C}$ ', 'B' and 'A' respectively of $b Q_{n}$-block. The output of this circuit is $m_{q}$. Again, $a_{n-3}$ and $a_{n-4}$ binary bits are connected into 'B' and ' $\mathrm{A}$ ' inputs of next $b Q_{n-1}$-block. 'C' input of this block is grounded i.e. fed to 0 volt. The output of this block is $m_{q-1}$. Other bits are put into other $b Q$-blocks $\left(b Q_{n-1}, \ldots, b Q_{1}, b Q_{0}\right.$ respectively) as the same way of $b Q_{n-1}$-block. MSB of this converted QSD number is only signed bit. So, if we look only 


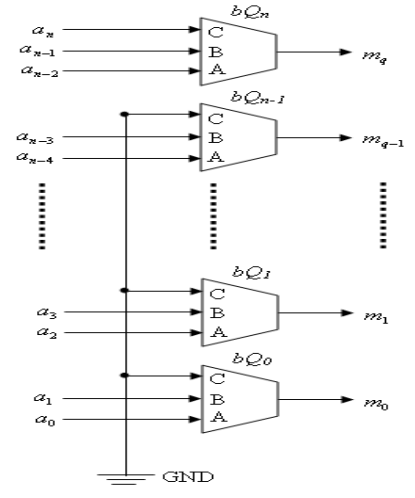

Figure 6: n-bit Binary Data to q-digit QSD Data Conversion Circuit

the MSB then, we can easily understand (without calculation) whether it is positive or negative. If the MSB of any QSD-data is one of $\overline{3}, \overline{2}, \overline{1}$ then, the data is negative. Otherwise it is positive.

\section{REFERENCES}

[1] T. Chattopadhyay and J.N. Roy, "All-optical multi-valued computing: the future challenges and opportunities", International conference on convergence of Optics and Electronics, (COE 11), March 26-27, Kolkata, Pp. 94-101, 2011, ISBN 978-81-906401-1-4.

[2] D.D. Givone, M.E. Liebler and R.P. Roesser, "A method of solution for multiple valued logic expression”, IEEE Trans. on computers, short notes, Pp. 464-467, April 1971.

[3] S. L. Hurst, "Multiple-Valued Logic-Its Status and its Future". IEEE Transactions computers, Vol. C-33, No. 12, Pp. 1160-1179, 1984.

[4] D. Etiemble, M. Israël, "Comparison of binary and Multivalued ICs according to VLSI criteria”, IEEE Computer, Pp. 28-42, April 1988.

[5] T. Chattopadhyay, G.K. Maity and Jitendra Nath Roy, "Designing of alloptical tri-state logic system with the help of optical nonlinear material", Journal of Nonlinear Optical Physics \& Materials, Vol. 17, No. 3, Pp.315-328, 2008.

[6] T. Chattopadhyay, "All-optical symmetric ternary logic gate”, Optics and Laser Technology, Vol. 42, Pp.1014-1021, 2010.

[7] C. Taraphdar, T. Chattopadhyay and J.N. Roy, "Designing of an alloptical scheme for single input Ternary logical operations", Optik International Journal for Light and Electron Optics, Vol. 122, No. 1, Pp. 33-36, 2011.

[8] T. Chattopadhyay, P. Bhowmik and J.N. Roy, "Polarization encoded optical N-valued inverter”, JOSA B, No. 29, No. 10, Pp. 2852-2860, 2012.

[9] M.A. Karim, A. Awwal, (1992). Optical Computing: an introduction. New York: John Wiley \& sons, Inc. Chap-6.

[10] H. Kerkhoff, M. Tervoert, "Multiple-Valued logic Charge-Coupled Devices”. IEEE Transactions computers, Vol. C-30, No. 9, Pp. 644-652, 1981.

[11] J. Mangin, K. Current, "Characteristics of prototype CMOS quaternary logic Encoder-Decoder circuits. IEEE Transactions on Computers”, Vol. C-35, No. 2, Pp. 157-161, 1986.

[12] M. Brilman, D. Etiemble, J.Oursel, P.Ttareau, “A 4-valued ECL encoder and decoder circuit". IEEE Journal of solid-state circuits, vol. SC-17, No. 3, Pp. 547-552, 1982.

[13] N. Shanbhag, D. Nagchoudhuri, R. Ferd, G. Visweswaran, "Quaternary Logic Circuits in 2-jum CMOS Technology”. IEEE Journal of solidstate circuits, Vol. 25, No. 3, Pp. 790-798, 1990.

[14] S. Han, S. Park, H. Seong, H. Kim, "A current-mode folding/ Interpolating CMOS analog to quaternary converter using binary to quaternary encoding block”. Proceeding of the $32^{\text {nd }}$ IEEE international symposium on multiple-valued logic (ISMVL'02), 0-7695-2831-7/07 2002.
[15] T. Chattopadhyay and J.N. Roy, "All-optical conversion scheme: binary to quaternary and quaternary to binary number". Optics \& Laser Technology, Vol. 41, Pp. 289-294, 2009.

[16] E.J. McCluskey, "Logic design of multivalued $I^{2} L$ logic circuits", IEEE Trans. Computers, Vol. C-28, Pp. 546-559, 1979.

[17] H.G. Kerkhoff and M.L. Tervoert, "Multivalued-valued logic charge couple devices”, IEEE Trans. Computers, Vol. C-30, Pp. 644-652, 1981.

[18] E.J. McCluskey, "Logic design of MOS ternary logic", Proc. $10^{\text {th }}$ International symp. Multiple-valued Logic, Pp. 1-5, 1980.

[19] A. Druzeta, Z.G. Vranesic and A.S. Sedra, "Application of multithreshold elements in realization of nany-valued logic networks", IEEE Trans. Computers, Vol. C-23, Pp. 1194-1198, 1984.

[20] S.J. Park, B.H. Yoon, K.S. Yoon and H.S. Kim, "Design of quaternary logic gate using double pass-transistor logic with neuron MOS down literal circuit”, Proceeding of the $34^{\text {nd }}$ IEEE international symposium on multiple-valued logic (ISMVL’04), Pp. 1-6, 2004.

[21] J.G. Tront and A.V. Thakar, "An analysis of FET based multiple-valued logic circuits”, Proc. 1982 Int. Symp. Multiple-Valued Logic, Pp. 69-76, 1982.

[22] C.R. Mingoto Jr, "A quaternary half-adder using current operation with bipolar transistor", Proceeding of the $36^{\text {nd }}$ IEEE international symposium on multiple-valued logic (ISMVL'06), Pp. 1-6, 2006.

[23] A. Tanabe, Y. Nakahara, A. Furukawa and T. Mogami, "A redundant multivalued logic for a 10-Gb/s CMOS demultiplexer IC”, IEEE journal of solid-state circuits, Vol.38, No.1, Pp.107-113, January 2003.

[24] S.R. Datla, M.A. Thornton, L. Hendrix and D. Henderson, "Quaternary addition circuits based on SUSLOC voltage-mode cells and modeling with Verilog”, Proceeding of the $39^{\text {nd }}$ IEEE international symposium on multiple-valued logic (ISMVL’09), Pp. 256-261, 2009.

[25] C.L. Lee, H.N. Chern, M.S. Liao and H.M. Wang, "On designing of 4valued memory with double-gate TFT”, IEEE conf., 0-8186-7118-1/95, Pp.187-192, 1995.

[26] S. Aragaki, T. Hanyu and T. Higuchi, "A multiple-valued contentaddressable memory using logic-value conversion and threshold functions”, IEEE Conf., 0195-623X/93, Pp.170-175, 1993.

[27] N. Abu-Khader and P. Siy, "Multiple-valued logic approach for a systolic $\mathrm{AB}^{2}$ circuit in Galois field", Proceeding of the $35^{\text {th }}$ IEEE international symposium on multiple-valued logic (ISMVL'05), 0195623X/05, Pp. 1-6, 2005.

[28] N. Abu-Khader and P. Siy, "Inversion/diversion in Galoies field using Multiple-valued logic", Proceeding of the $37^{\text {th }}$ IEEE international symposium on multiple-valued logic (ISMVL'07), 0-7695-2831-7/07, Pp. 1-6, 2007.

[29] M.H.A. Khan, M.A. Perkowski, M.R. Khan and P. Kerntopf, "Multioutput Galoies field sum of products synthesis with new quantum cascades”, Proc. $33^{\text {rd }}$ IEEE Int. symp. on multiple-valued logic, Tokyo, Pp.41-47, 2003.

[30] M.H.A. Khan, M.A. Perkowski, M.R. Khan and P. Kerntopf, "Ternary GFSOP minimization using kronecker decision diagrams and their synthesis with quantum cascades", J. of Mult.-valued logic \& soft computing, Vol. 11, Pp.567-602, 2005.

[31] A.A.S. Awwal, J. Ahmed, "Fast Carry free Adder Design Using QSD Number System”. IEEE, CH3306-8/93/0000-1085, Pp. 1085-1088, 1993.

[32] W. Clark, J. Lian, "On arithmetic weight for general radix representation of integers”. IEEE Trans. Inform. Theory, Vol. 19, Pp. 823-826, 1973.

[33] A.Cherri, "Canonical Quaternary arithmetic based on optical contentaddressable memory (CAM)”. IEEE, CH35934-96/0000-0655, pp. 655659, 1996.

[34] T. Chattopadhyay, J.N. Roy, "Easy conversion technique of binary to quaternary signed digit and vice versa.” Physics Express, Vol. 1, No. 3, Pp. 165-174, 2011.

[35] J. Millman, C. Halkias, Integrated Electronics: Analog and digital circuits and systems. New York: McGraw-Hill, Inc. Chap-17. 1972.

[36] R.P.Jain. Modern Digital Electronics ( $3^{\text {rd }}$ Edition). New Delhi: Tata McGraw-Hill Publishing Company Limited. Chap-10 [Sec.10.2.1]. 2003.

[37] Vasundara Patel, K.S. Gurumurthy, "Arithmetic operations in multivalued logic.” International Journal of VLSI Design \& Communication Systems (VLSICS), Vol. 1, No. 1, March 2010.

[38] Nabi Abu-Khader, Pepe Siy, "Systolic product sum circuit for GF ((22)m ) using neuron MOSFET”, INTEGRATION, The VLSI journal, Vol. 39, Pp. 29 - 47, 2005.

[39] K. Ogawa, T. Shibata, T. Ohmi, M. Takatsu, and N. Yokoyama, "Multiple-Input Neuron MOS Operational Amplifier for Voltage-Mode 
Multivalued Full Adders”, IEEE transactions on circuits and systemsII: analog and digital signal processing, Vol. 45, No. 9, Pp. 1307-1311, September 1998.

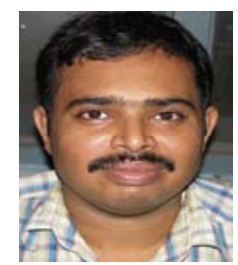

Tanay Chattopadhyay was born in Kolkata, West Bengal, India on January 15, 1977. He received M.Sc degree in physics with special paper electronics from university of Calcutta on 2002. He also received diploma in computer hardware maintenance and Networking in 2006. On 2012 he has got P.hD degree from West Bengal University of Technology in Physics.

He is currently working on Kolaghat Thermal Power Station, West Bengal as Jr. Manager (power station). His present research interests include optical logic based information processing, multiple-valued logic, laser and nonlinear optics, optics communication. He has more than eighty papers published /accepted in international journals and symposia proceedings and two book chapters. He is also a reviewer of several reputed International Journals viz. Elsevier, SPIE, Springer, IEEE, OSA etc. Dr. Chattopadhyay joins as an Editorial Board Member in Global Journal of Physics Express on July 2010. He is selected for Who's Who in the World in 2012 and 100 Top professions of 2012 in British International Biographical center.

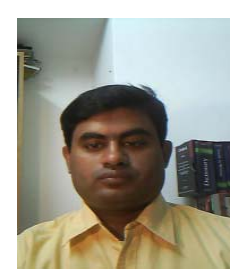

Tamal Sarkar was born in Balurghat, West Bengal, India on September, 18, 1977. He received Bachelor Degree with Physics Honors in 1998 from North Bengal University and Master Degree (Physics) in year 2000.

$\mathrm{He}$ is associated with University Science instrumentation centre, North Bengal University as Technical Officer since 2007. His area of interest includes High Energy Astrophysics, X-Ray Astronomy, Quantum Computing and Theoretical Opto-electronics. He has published few research articles in National and International Journals in above mentioned field. Apart from it, he is a guest faculty for the course of Post Graduate Diploma in Remote Sensing and GIS of Centre of Remote Sensing, North Bengal University. He has also worked as member of Expert Committees/ Tender Committees for University of North Bengal. He is also a member of FOSET, Kolkata. 